\title{
ON THE ORDER OF MAGNITUDE OF FOURIER TRANSFORM
}

\section{BHIKHA LILA GHODADRA AND VANDA FÜLÖP}

Abstract. For a Lebesgue integrable complex-valued function $f$ defined on $\mathbb{R}$, let $\hat{f}$ be its Fourier transform. The Riemann-Lebesgue lemma says that $\hat{f}(t) \rightarrow 0$ as $|t| \rightarrow \infty$. But in general, there is no definite rate at which the Fourier transform tends to zero. In fact, the Fourier transform of an integrable function can tend to zero as slowly as we wish. Therefore, it is interesting to know for functions of which subclasses of $L^{1}(\mathbb{R})$ there is a definite rate at which the Fourier transform tends to zero. In this paper, we determine this rate for functions of bounded variation on $\mathbb{R}$. We also determine such rate of Fourier transform for functions of bounded variation in the sense of Vitali defined on $\mathbb{R}^{N}(N \in \mathbb{N})$.

Mathematics subject classification (2010): 42A38, 42B10, 26A12, 26A45, 26B30, $26 \mathrm{D} 15$.

Keywords and phrases: Fourier transform, function of bounded variation over $\mathbb{R}$, function of bounded variation over $\mathbb{R}^{2}$, function of bounded variation over $\mathbb{R}^{N}$, order of magnitude.

\section{REFERENCES}

[1] C. R. Adams and J. A. Clarkson, Properties of functions $f(x, y)$ of bounded variation, Trans. Amer. Math. Soc., 36, (1934), 711-730.

[2] J. A. Clarkson And C. R. Adams, On definitions of bounded variations for functions of two variables, Trans. Amer. Math. Soc., 35, (1933), 824-854.

[3] R. E. Edwards, Fourier Series, A Modern Introduction, Volume 1, Second Edition, Springer-Verlag, New York, Heidelberg, Berlin, 1979.

[4] V. FÜLÖP AND F. MÓRICZ, Order of magnitude of multiple Fourier coefficients of functions of bounded variation, Acta Math. Hungar., 104, (2004), 95-104.

[5] B. L. GHODADRA, Order of magnitude of multiple Fourier coefficients of functions of bounded pvariation, Acta Math. Hungar., 128, (2010), 328-343.

[6] S. R. Ghorpade And B. V. Limaye, A Course in Multivariable Calculus and Analysis, Springer, 2009.

[7] R. R. Goldberg, Fourier Transforms, Cambridge University Press, 1961.

[8] G. H. HARDY, On double Fourier series, Quart. J. Math., 371, (1906), 53-79.

[9] E. HewitT AND K. A. Ross, Abstract Harmonic Analysis II, Springer-Verlag, Berlin, 1970.

[10] E. W. HobSON, The theory of functions of a real variable and the theory of Fourier's series, Vol. I, Dover Publications Inc., New York, 1927.

[11] H. LEBESGUE, Sur la représentation trigonometrique approchée des fonctions satisfaisant a une condition de Lipschitz, Bull. Soc. Math. France, 38, (1910), 184-210.

[12] F. MóRICZ, Order of magnitude of double Fourier coefficients of functions of bounded variation, Analysis, 22, (2002), 335-345.

[13] F. MÓRICZ, Pointwise convergence of double Fourier integrals of functions of bounded variation over $\mathbb{R}^{2}$, Submitted.

[14] I. P. NATANSON, Theory of functions of a real variable, Vol. I, New York, 1964.

[15] E. M. STEIN And G. Weiss, Introduction to Fourier Analysis on Euclidean Spaces, Pinceton, New Jersey, 1971.

[16] M. TAibleson, Fourier coefficients of functions of bounded variation, Proc. Amer. Math. Soc., 18, (1967), 766.

[17] A. Zygmund, Trigonometric series, Third Edition, Vol. I and II combined, Cambridge University Press, 2002. 